\title{
Metagenomics reveals functional synergy and novel polysaccharide utilization loci in the Castor canadensis fecal microbiome
}

\author{
Zachary Armstrong $\mathbb{1}^{1}{ }^{1} \cdot$ Keith Mewis $^{1} \cdot$ Feng Liu $^{2} \cdot$ Connor Morgan-Lang ${ }^{3} \cdot$ Melanie Scofield $^{3}$ - Evan Durno ${ }^{3}$. \\ Hong Ming Chen ${ }^{2} \cdot$ Kevin Mehr ${ }^{1} \cdot$ Stephen G. Withers ${ }^{1,2,4,5,6} \cdot$ Steven J. Hallam $\mathbb{1}^{1,3,6,7,8}$
}

Received: 23 August 2017 / Revised: 15 April 2018 / Accepted: 1 June 2018 / Published online: 16 July 2018

(c) The Author(s) 2018. This article is published with open access

\begin{abstract}
The North American beaver (Castor canadensis) has long been considered an engineering marvel, transforming landscapes and shaping biological diversity through its dam building behavior. While the beaver possesses conspicuous morphological features uniquely adapted for the use of woody plants as construction materials and dietary staples, relatively little is known about the specialized microorganisms inhabiting the beaver gastrointestinal tract and their functional roles in determining host nutrition. Here we use a combination of shotgun metagenomics, functional screening and carbohydrate biochemistry to chart the community structure and metabolic power of the beaver fecal microbiome. We relate this information to the metabolic capacity of other wood feeding and hindgut fermenting organisms and profile the functional repertoire of glycoside hydrolase $(\mathrm{GH})$ families distributed among and between population genome bins. Metagenomic screening revealed novel mechanisms of xylan oligomer degradation involving GH43 enzymes from uncharacterized subfamilies and divergent polysaccharide utilization loci, indicating the potential for synergistic biomass deconstruction. Together, these results open a functional metagenomic window on less conspicuous adaptations enabling the beaver microbiome to efficiently convert woody plants into host nutrition and point toward rational design of enhanced enzyme mixtures for biorefining process streams.
\end{abstract}

\section{Introduction}

Microbial communities inhabiting the mammalian digestive tract, gut microbiomes, affect host health and mediate essential services including dietary access to recalcitrant

These authors contributed equally: Zachary Armstrong and Keith Mewis.

Electronic supplementary material The online version of this article (https://doi.org/10.1038/s41396-018-0215-9) contains supplementary material, which is available to authorized users.

Steven J. Hallam

shallam@mail.ubc.ca

1 Genome Science and Technology Program, University of British Columbia, 2329 West Mall, Vancouver, BC V6T 1Z4, Canada

2 Department of Chemistry, University of British Columbia, 2036 Main Mall, Vancouver V6T 1Z1 BC, Canada

3 Department of Microbiology \& Immunology, University of British Columbia, Vancouver V6T 1Z1 BC, Canada

4 Centre for High-Throughput Biology, University of British glycans such as starches and fibers [1]. With respect to digestion, the taxonomic composition of these communities correlates with host diet and nutrient acquisition strategies across different mammalian lineages [2]. Multiple studies indicate that mammalian gut microbiomes consist of specialized communities that respond to complex glycans derived from specific dietary sources such as lignocellulosic biomass and release products that can be absorbed into the digestive tract [2-6]. Consistent with these observations, the digestive tracts of herbivores and wood-feeding (xylotrophic) organisms harbor microbial communities enriched in genes or gene cassettes encoding the corresponding

Columbia, Vancouver V6T 1 Z3 BC, Canada

5 Department of Biochemistry and Molecular Biology, University of British Columbia, Vancouver V6T 1Z3 BC, Canada

6 ECOSCOPE Training Program, University of British Columbia, Vancouver V6T 1 Z3 BC, Canada

7 Graduate Program in Bioinformatics, University of British Columbia, Vancouver V6T 1Z4 BC, Canada

8 Peter Wall Institute for Advanced Studies, University of British Columbia, Vancouver V6T 1Z1, Canada 
biocatalysts and polysaccharide utilization systems [7-12]. These communities provide a frame of reference for understanding how lignocellulosic biomass is converted into dietary macronutrients, as well as a deep reservoir of genomic information with potential biotechnological applications [13].

The North American beaver, Castor canadensis, provides a useful animal model for the study of xylotrophic microbiomes, as its diet is largely composed of bark, shoots, leaves, and other fibers from hardwood deciduous trees such as poplar, aspen, and cottonwood, which have commercial value in the forestry sector [14, 15]. Hardwoods such as poplar typically have a total polysaccharide content comprising $60-80 \%$ of the dry mass of the wood [16]. Some $35-50 \%$ of this dry mass consists of cellulose followed by hemicellulose (20\% primarily glucuronoxylan) and pectin. Previous studies have shown that beavers are capable of digesting up to $32 \%$ of the available cellulose in consumed hardwoods [17]. However little is known about the utilization of the hemicellulose component by the beaver microbiome, and much less about the enzyme repertoire effecting its deconstruction.

Gruninger and colleagues recently used small subunit ribosomal RNA (SSU rRNA) gene sequencing to profile the microbial community structure of beaver cecum and feces, indicating a typical mammalian hindgut community dominated by Bacteroidetes and Firmicutes [18]. As a hindgut fermenter, commensal microbes in the lower digestive tract of the beaver are expected to mediate the degradation and fermentation of complex sugars to provide short chain fatty acids that provision host nutrition [19]. Given that the proclivity of beavers to consume wood differentiates them from other hindgut fermenting herbivores, several questions arise from the initial microbiome study: At what taxonomic level does the beaver microbiome differ from other xylotrophic and hindgut fermenting organisms? Does the microbiome encode specialized genes or gene cassettes mediating complete conversion of lignocellulosic biomass? Are some components of the lignocellulose targeted for digestion more than others? To what extent are these functions distributed at the population and community levels? Could analysis of these differences reveal new insight into sequential biomass deconstruction of woodbased fibers transferrable to industrial process streams? To begin answering these questions, we used a combination of SSU rRNA gene sequencing, shotgun metagenomics and functional screening to evaluate the community structure and metabolic potential of the beaver microbiome in relation to other wood-feeding organisms and to recover activities mediating lignocellulosic biomass deconstruction.

\section{Results}

To profile the microbial community composition of the beaver fecal microbiome, we performed 454 pyrotag sequencing of the V6-V8 region of the SSU rRNA gene with three-domain resolution on composite fecal samples from 2 captive beavers. A total of 12,579 rRNA pyrotag sequences, recovered from the fecal sample, were clustered with a $97 \%$ similarity cut-off into 404 operational taxonomic units (OTUs) after singleton removal. Of the OTUs identified, only two could not be affiliated with described microbial taxa based on homology to sequences in the Silva database [20] (Fig. 1). One of these OTUs showed 99\% identity to the Castor canadensis mitochondrial DNA sequence [21], while the other had at most $75 \%$ sequence identity to SSU rRNA sequences from uncultured bacteria. The majority of sequences were affiliated with the bacterial phyla Firmicutes (214 OTUs, 58.4\%) and Bacteroidetes (93 OTUs, 24.4\%). Within the Firmicutes, 200 OTUs were affiliated with the class Clostridia (55.9\%), with 143 OTUs (43.6\%) affiliated with the family Lachnospiraceae, which is known to harbor xylanotrophic, butyric acid producing members [22]. Within the Bacteroidetes, 68 of the 93 OTUs $(21.3 \%)$ were affiliated with the class Bacteroidia, with 39 OTUs (15.5\%) affiliated with the uncultivated S24-7 group. In a recent cultivationdependent study, S24-7 comprised approximately $4 \%$ of the beaver fecal microbiome prior to methanogenic enrichment on different lignocellulosic biomass substrates [23]. Overall, these results are consistent with the observations of Gruninger and colleagues, although the proportions of Firmicutes and Bacteroidetes were not identical [18]. Additionally, the dominance of Firmicutes and Bacteroidetes is also seen in other wild rodents [24, 25]. As a high proportion of identified OTUs lacked cultured representatives (354 of 370 bacterial OTUs) specific metabolic roles could not be inferred with confidence. To this end

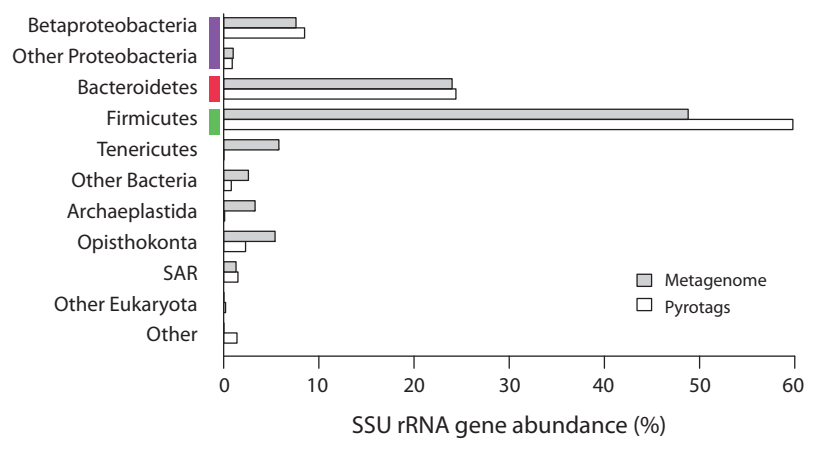

Fig. 1 Beaver fecal community composition. The relative abundance of 16s rDNA genes found in the metagenome are compared to those identified by pyrotags. Both methods reveal a metagenome dominated by Firmicutes (green), Bacteroidetes (red) and Proteobacteria (purple) phyla 
we used shotgun metagenome sequencing to predict metabolic functions encoded in the beaver microbiome.

Shotgun metagenomic sequencing was conducted on the 454 platform from the same fecal DNA preparations used in SSU rRNA gene pyrotag analysis resulting in the production of 469.2 million base pairs (Mbp) of total sequence information $(616,811$ reads with average length $761 \mathrm{bp})$. Raw sequences were trimmed to Q30 quality score using prinseq lite + [26] and assembled using MIRA [27], resulting in 75,523 contigs with an N50 of $1,787 \mathrm{bp}$ and $130.5 \mathrm{Mbp}$ of consensus sequence. One of these assembled contigs (beaver_rep_c845) had 99\% identity with the $16 \mathrm{~kb}$ beaver mitochondria assembled by Horn and colleagues providing an additional reference for host population studies [21].

To explore potential bias in community structure based on pyrotag analysis, we examined SSU rRNA gene sequences recovered from the metagenome by comparing unassembled reads to the Silva SSU database using MetaPathways [28]. Of the unassembled reads 1,812 were annotated as having SSU rRNA genes (Supplementary Table 1). The majority of these sequences were affiliated with either Firmicutes (890) or Bacteriodetes (438), consistent with pyrotag results (Fig. 1). A notable exception was the relative abundance of Tenericutes within the metagenome, which was greater than that seen in the pyrotag data (percentages of $5.8 \%$ and $0.02 \%$ respectively). This may be due to amplification bias, as has previously been observed for Mycoplasma, the dominant Tenericutes genus identified in the beaver fecal metagenome [29]. In addition to bacteria, we detected SSU rRNA gene sequences affiliated with Archaeplastida (predominant class Liliopsida) and Opisthokonta (predominant class Insecta) at low abundance. The presence of these eukaryotic taxa in the beaver microbiome could reflect captive dietary intake or colonization post defecation.

Given the similarities between the taxonomic composition of the beaver fecal microbiome and other hindgut fermenters we initially looked for differences in carbohydrate active enzymes (CAZymes). Unassembled reads from the metagenome were queried against the CAZy database [30] using MetaPathways [28] and compared to 37 other mammalian species [2, 31, 32]. Because older datasets tended to have reduced and variable coverage per sample, unassembled metagenomic reads were compared using a variance stabilizing transformation (VST) [33]. CAZymes (all classes) constituted $3.85 \%$ of predicted genes within the beaver fecal microbiome compared to $2.89-6.95 \%$ for other organisms (Supplementary Table 2). Consistent with previous reports, CAZymes formed distinct clusters with glycoside hydrolase $(\mathrm{GH})$ families encoding cellulases, hemicellulases, and pectinases overrepresented in herbivores, and families encoding glycosaminoglycanases overrepresented in carnivores [2, 5] (Supplementary Fig. 1). Overall, the beaver CAZyme profile was most similar to those of hindgut-fermenting herbivores.

To determine the metabolic context of GH families in the beaver fecal microbiome we reconstructed metabolic pathways on open reading frames (ORFs) predicted on assembled contigs and annotated using MetaPathways [28]. Of the 48,959 ORFs mapped to KEGG pathways, 9514 or $19.4 \%$ were associated with carbohydrate metabolism (Supplementary Table 3). In particular, ORFs encoding beta-glucosidase (bglX: K05349) and beta-galactosidase (lacZ: K01190), were similar in abundance to those of other mammals $(b g l X$ mean $=0.313 \pm 0.14 \%$, beaver $=0.347 \%$; lac Z mean $=0.342 \pm 0.13 \%$, beaver $=0.368 \%$ ).

Based on observed functional similarities between CAZymes and KEGG pathways present in the beaver fecal microbiome and other hindgut fermenting herbivores, we wondered if functional organization of these genes at the population or community levels could in part explain the capacity of the beaver to digest wood fibers efficiently. Contigs and functionally identified fosmids (described below) were binned into population genomes using MaxBin 2.0 [34] which were refined using the Anvi'o metagenomics workflow [35]. This binning produced 8 medium-quality (completeness $>50 \%$ and contamination $<10 \%$ ) and 22 lowquality metagenome-assembled genomes (MAGs) [36]. Together these bins accounted for $6.2 \%$ of the assembled metagenome (Supplementary Table 4). Further analysis was confined to the medium-quality bins. We then used PhyloSift to assign taxonomy to these bins [37]. Two bins were assigned to the Bacteroidetes phylum (Bins 1 and 2), five to the order Clostridiales (3, 5, 7, 8, 13 and 16) and one to an undefined genus within the Burkholderiales order (Bin 6). Three Clostridiales bins could be further classified beyond the order level, with one classified at the Genus level as a Flavobacterium (Bin8), and two classified at the species level as Coprococcus sp. ART55/1 (Bin13) and Eubacterium eligens (Bin16). This species-level assignment was cross-validated using average nucleotide identity (ANI) to known isolate genomes, revealing that Bin13 had $97.5 \%$ ANI to the Coprococcus sp. ART55/1 genome, while Bin16 had 95.6\% ANI to Eubacterium eligens strain ATCC 27750. The strain Coprococcus sp. ART55/1, which belongs to the family Lachnospiraceae, is likely involved in the generation of fermentation products within the beaver gut as it has previously been shown to produce butyrate [38].

The abundance of plant carbohydrate active $\mathrm{GH}$ families within the identified population genome bins was then investigated (Supplementary Table 5). All medium-quality bins exhibited incomplete enzyme repertoires involved in lignocellulosic biomass deconstruction consistent with combinatorial substrate conversion processes. One set of 

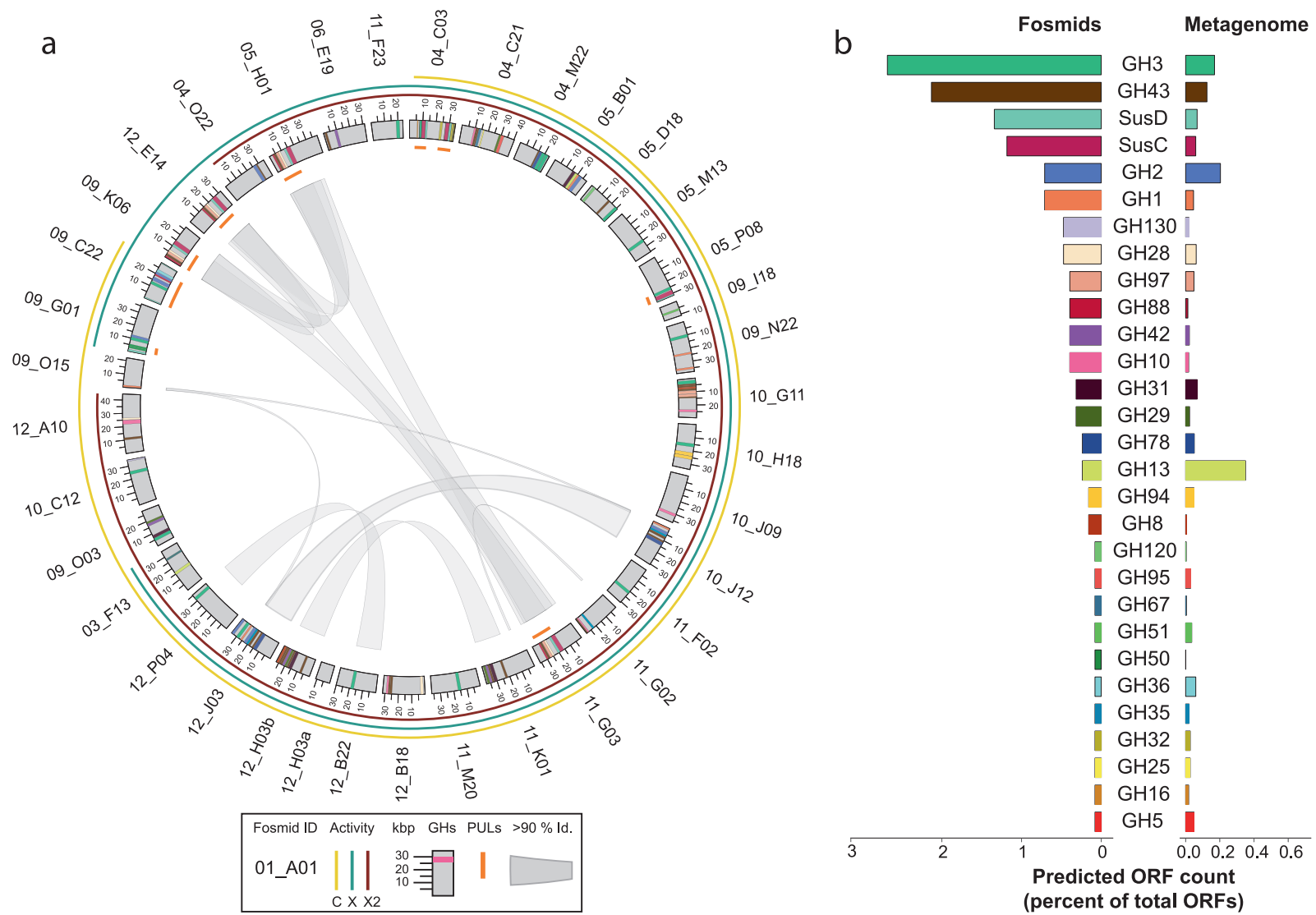

Fig. 2 Fosmids identified from high throughput screening. a Schematic representing the identified fosmids, gene presence and similarity. Gray bars represent each fosmid and are proportional to their length. Fosmids sharing $100 \%$ identity with another fosmid were removed as duplicates. Connections in the center represent areas of $90 \%$ or greater nucleotide identity between fosmids as identified by BLASTN. Inner track represents the locations of identified PULs. Outer colored track represents activities identified for each fosmid from functional

bins $(1,13,16)$ contained a wide range of genes encoding hemicellulose degrading enzymes (average $2.1 \%$ of all ORFs), but lacked genes encoding enzymes necessary for glucuronoxylan debranching (GH families 67 and 115). Cellulose degradation appeared to be chiefly performed by bins $1,2,7$, and 13 , as these were the only bins to contain GH families with endo-cellulase activity. Three of the eight bins appeared to target pectins $(1,7$, and 16) with Bin16, the Eubacterium eligens bin, having the most extensive array of pectin targeting genes. This fits well with the previous observations of pectin degradation by this species [39]. The Coprococcus bin (Bin13) contained plant biomass targeting GH and CE families (GH43, GH95, CE4) that were absent in the Coprococcus sp. ART55/1 genome, suggesting expanded ability to degrade carbohydrates by the strain found in the beaver feces. The remaining bins $(2,3$, and 8$)$ appeared to have a comparatively reduced capacity to degrade lignocellulosic biomass, but contained multiple enzymes for starch or glycogen degradation screening (C: CMU-cellobiose, X: CMU-xylose, X2: CMU-xylobiose). Colored bars within each fosmid represent $\mathrm{GH}$ domains as predicted by BLASTP against the CAZy database. b Histogram displays color encoding of $\mathrm{GH}$ gene families as well as abundance of each family in the complete fosmid dataset compared to the abundance of the same gene families in the unassembled metagenomic dataset. The abundance relative to the total number of ORFs is shown in parentheses

as well as simple glucosides and gluco-oligosaccharides present within the beaver diet or liberated by the endoacting enzymes of co-occurring microorganisms.

While this analysis provided more specific information regarding metabolic potential of observed community members, specific CAZyme functions were inferred solely on the basis of sequence homology. Although enzyme activity often correlates with CAZy family, new activities are often identified within existing families, such as those identified by Ndeh et al. [40]. To better validate gene models we conducted functional screening to recover active CAZymes from large insert (fosmid) clones.

A fosmid library containing over 4,500 clones was constructed from the same DNA used in shotgun metagenome sequencing using the pCC1 copy control system expressed in E. coli EPI300. Activity assays were based on methods described by Mewis and colleagues [41, 42] but instead of chromogenic substrates we utilized cellobioside, xyloside, and xylobioside fluorogenic substrates bearing the 
6-chloro-7-hydroxy-4-methylcoumarin aglycone, resulting in greater sensitivity with improved signal to noise ratio [43]. We combined the three substrates in a multiplex format to reduce both the time required and materials costs (Supplementary Fig. 2). Multiplex screening identified 51 fosmids that hydrolyzed at least one of the three fluorogenic substrates (z-score > 3); a hit rate of 1 in 88 (Supplementary Fig. 3). Substrate specificities of active clones recovered in multiplex screening were further assessed against a panel of nine separate fluorogenic substrates (Supplementary Fig. 4). A majority of clones were most active against either $\beta$ glucosides or $\beta$-xylosides However, six clones displayed higher activities against alternative substrates including arabinose (06_E19,09_O03, 09_O15), galactose (10_J12), lactose (9_I18), and mannose (5_B01). This suggests that either the active enzymes encoded on these fosmids possess broad substrate specificities, or that multiple functions are encoded and expressed from individual clones consistent with gene cassettes e.g., cellulosomes or polysaccharide utilization loci (PULs) involved in the extracellular deconstruction of insoluble biomass [44] and the utilization of soluble carbohydrates within the cell respectively [45].

To identify individual genes or gene cassettes mediating substrate conversion we fully sequenced the 51 active clones. Reads were assembled using ABySS [46] and ORFs predicted and annotated using the MetaPathways pipeline [28]. Comparison between sequences identified 38 nonredundant clones based on $>95 \%$ similarity across $>90 \%$ of insert length (Fig. 2a). The reads mapped per kilobase of fosmid per million mapped reads (RPKM) from the metagenome was highly variable between fosmids, with an average value of $8.7 \pm 13.0$ per fosmid; a maximum of 81.9 and a minimum of 0.1 (Supplementary Table 6). Few fosmids had greater than $90 \%$ metagenome coverage (04_O22, 05_H01, 12_E14) with two fosmids (09_N21, 09_N22) recruiting less than $5 \%$ of their total sequence length (Supplementary Table 6). LCA assignment of 1,267 predicted ORFs indicated that 13 active clones were affiliated with Firmicutes and 38 were affiliated with Bacteroidetes donor genotypes (Supplementary Fig. 5). When queried against the medium-quality metagenomic bins, 5 active clones had $>97 \%$ identity to a contig larger than $1 \mathrm{~kb}$ (Supplementary Table 6). This number includes 04_O22, the only fosmid to be assigned to a medium-quality bin. The four remaining fosmids (05_H01, 09_K06, 11_G03, and 12_E14) all aligned with over $75 \%$ of their lengths to Bin 1.

Queries of sequenced fosmids against the CAZy database identified $135 \mathrm{GH}$ genes from $28 \mathrm{GH}$ families encompassing $11.13 \%$ of the annotated ORFs (Fig. 2b). Unexpectedly, active fosmids harbored only $5 \mathrm{GH}$ genes from families with annotated cellobiohydrolases or endoglucanases, one each from GH families 5 and 51, and three from GH8 with none from the cellulolytic GH families $6,7,9,12,26,44$,
$45,48,74$, or 124 . As the metagenome encodes for a total of 1,085 cellulases from 9 of 14 cellulase families $(0.15 \%$ of all predicted metagenome ORFs) it may be that we have only captured the most abundant taxa within the fosmid library and that cellulose is being degraded by rarer taxa within the community. In contrast, there was an abundance of genes encoding hemicellulose converting enzymes, particularly xylan. The most abundant GH family recovered was GH3, which contains both $\beta$-xylosidases and $\beta$-glucosidases. This was followed in abundance by GH43, a family containing both $\beta$-xylosidases and xylanases [47]. Within the unassembled metagenome, 115 GH43 genes ( $0.015 \%$ of all metagenomic genes) from 23 subfamilies were identified, the second highest diversity of GH43 subfamilies seen in any of the mammalian metagenomes analyzed (Supplementary Table 7). A total of 27 GH43 genes (1.8\% of fosmid genes) from 10 subfamilies were identified on active clones (Table 1). Three GH43 genes, 12_H03 (two genes - subfamilies 2 and 7) and 12_J03 (subfamily 28), belonged to subfamilies containing

Table 1 GH43 subfamilies identified on functionally active fosmids

\begin{tabular}{|c|c|c|}
\hline Fosmid & ORF & GH43 subfamily \\
\hline Beaver_04_C21 & 10 & 12 \\
\hline Beaver_04_C21 & 11 & 1 \\
\hline Beaver_04_M22 & 11 & 24 \\
\hline Beaver_04_O22 & 25 & 19 \\
\hline Beaver_05_D18 & 17 & 11 \\
\hline Beaver_05_H01 & 3 & 10 \\
\hline Beaver_06_E19 & 1 & 11 \\
\hline Beaver_09_K06 & 1 & 10 \\
\hline Beaver_10_G11 & 3 & 2 \\
\hline Beaver_10_G11 & 4 & 29 \\
\hline Beaver_10_G11 & 8 & 29 \\
\hline Beaver_10_J12 & 4 & 28 \\
\hline Beaver_10_J12 & 7 & 24 \\
\hline Beaver_11_G02 & 25 & 1 \\
\hline Beaver_11_G03 & 16 & 10 \\
\hline Beaver_11_K01 & 12 & 12 \\
\hline Beaver_11_K01 & 19 & 29 \\
\hline Beaver_12_A10 & 9 & 12 \\
\hline Beaver_12_B18 & 18 & 11 \\
\hline Beaver_12_B18 & 19 & 12 \\
\hline Beaver_12_E14 & 11 & 10 \\
\hline Beaver_12_H03 & 10 & 29 \\
\hline Beaver_12_H03 & 12 & 7 \\
\hline Beaver_12_H03 & 13 & 2 \\
\hline Beaver_12_H03 & 3 & 12 \\
\hline Beaver_12_J03 & 15 & 24 \\
\hline Beaver_12_J03 & 18 & 28 \\
\hline
\end{tabular}


no previously characterized members thus were of unknown specificities. Genes encoding xylan side-chain removing enzymes ( $\alpha$-glucuronidase from GH67; $\alpha$-galactosidases from GH 28 and 97) were also present. Interestingly, we identified a number of genes encoding multiple $\mathrm{GH}$ domains (Supplementary Fig. 6). Four of these encoded predicted endo-acting and exo-acting domains. These include 4_C21-10 and 12_B18-19 which contain both GH43 and GH10 domains and are likely involved in xylan degradation, as well as 10_G11-03 and 12_H03-13, both of which contain GH43 and GH8 domains consistent with a role in xyloglucan or xylan degradation. The presence of both endo- and exo-glycosidase domains within the same protein can lead to synergism in efficient deconstruction of polysaccharides, as has been observed previously [48].

To better understand the substrate specificities and activities of the enzymes present, we focused our attention on the uncharacterized GH43 subfamilies identified by functional screening. To this end we generated constructs that were used to overexpress and purify recombinant proteins (12_H03-13 from subfamily 2, 12_H03-12 from subfamily 7, and 12_J03-18 from subfamily 28). Since 12_H03-13, also contained a GH8 domain, we created two additional constructs in which the GH8 and GH43 domains were inactivated independently by mutation of the catalytic acid residue (GH8 domain mutant H03-13_E507A and GH43 domain mutant H03-13_E209A). Of the three wildtype enzymes, two had detectable cleavage activity on monosaccharide aryl glycosides. Both 12_H03-13 and 12_J03-18 cleaved CMU-xyloside, with the specificity constant of the 12_H03-13 wild-type enzyme being the same, within error, as that of the H03_E507A variant in which the GH8 activity was abated (Table 2), indicating that it was the GH43 domain that was responsible for the xylosidase activity. Surprisingly, none of the enzymes cleaved any of the other aryl glycosides tested (Supplementary Table 8), reinforcing the utility of these inherently more reactive chlorocoumarin glycosides for detection of previously unknown activities.

The activities of enzymes 12_H03-12, 12_H03-13 and its variants were also tested on a set of arabinoxylan oligosaccharides. This revealed a synergistic degradation mechanism in which the GH43 domain of 12_H03-13 (subfamily 2) releases undecorated xylose from the

Table 2 Kinetic parameters determined for purified GH43 enzymes with 6-chloro-4-methylumbelliferyl $\beta$-D-xyloside

\begin{tabular}{llll}
\hline Enzyme & $\mathrm{K}_{\mathrm{M}}(\mathrm{mM})$ & $\mathrm{k}_{\mathrm{cat}}\left(\mathrm{s}^{-1}\right)$ & $\mathrm{k}_{\text {cat }} \mathrm{K}_{\mathrm{M}}\left(\mathrm{s}^{-1} \mathrm{mM}^{-1}\right)$ \\
\hline 12_J03-18 & $0.48 \pm .06$ & $0.22 \pm .02$ & $0.45 \pm .09$ \\
12_H03-13 WT & $0.19 \pm .03$ & $0.80 \pm .06$ & $4.2 \pm 0.7$ \\
12_H03-13_E507A & $0.14 \pm .01$ & $0.73 \pm .02$ & $5.2 \pm 0.4$ \\
\hline
\end{tabular}

non-reducing end of the oligosaccharides while the GH8 domain of 12_H03-13 (a reducing end xylose-releasing exo-oligoxylanase $[\operatorname{Rex}]$ ) releases xylose from the reducing end of decorated oligosaccharides (Fig. 3). The activity displayed by 12_H03-13 is further complemented by GH43 12_H03-12 (subfamily 7), which cleaves $\alpha-1-3$-linked arabinose decorations from arabinoxylans, releasing arabinose and xylobiose. This activity is only observed in the presence of 12_H03-13. This establishes the intriguing possibility that 12_H03-12 is activated by 12_H03-13. The xylobiose generated by these two enzymes appears to be resistant to further degradation by these enzymes. As GH8 Rex genes typically require at least a trisaccharide for activity, this domain is not expected to hydrolyze xylobiose. The GH43 domain of 12_H03-13 was expected to further degrade xylobiose, yet this is not the case, suggesting that the presence of an arabinose sidechain may be important for the xylosidase activity of this domain. This represents, to our knowledge, the first multi-domain protein containing both a GH43 and GH8 domain to be characterized and the first description of how these two domains function synergistically on arabinoxylan oligosaccharides converting them into arabinose and xylobiose. Collectively these results illuminate substrate specificity and activity of GH43 subfamilies 2, 7, and 28 within the context of the beaver fecal microbiome with direct relevance to lignocellulosic biomass conversion and host nutrition.

In addition to providing a route toward functional validation of predicted $\mathrm{GH}$ genes, active clone sequences contained information about the structural organization of $\mathrm{GH}$ gene cassettes. This has revealed several GH gene clusters that appear to target plant cell wall hemicelluloses (Fig. 4a). The fosmid 04_C03 contains a motif (GH16 and GH3 adjacent to SusC and SusD-like proteins) with synteny to a PUL recently shown to be active against mixed-linkage glucans [49]. Several fosmids (04_C21, 10_G11, 11_G02, 12_H03, and 11_K01) also appear to target xylans. The four fosmids 04_C21, 11_G02, 12_H03, and 11_K01 all harbor GH10 genes, which often act as endo-xylanases, and GH43s which may have exoxylanase activity. Furthermore, Fosmid 04_C21 contains a motif (GH10-GH43 (GH43 subfamily 12) protein followed by an additional GH43 (subfamily 1) and a GH67 with synteny to a gene cluster identified in Bacteroides intestinalis [50]. The GH10-GH43 homolog from $B$. intestinalis has endoxylanase and arabinofuranosidase activity, which is able to release xylose, xylo-oligosaccharides and arabinose from arabinoxylans [50]. Although Fosmid 10_G11 lacks a GH10 it does contain a two domain GH43-GH8 gene, which we speculate may have similar activity to H03-13 in targetting arabinoxylan oligomers. The presence of a GH29 (a family of $\alpha$-L-fucosidases), GH42 (a family cotaining $\beta$-galactosidases) and $\mathrm{GH} 31$ (a family containing 
a

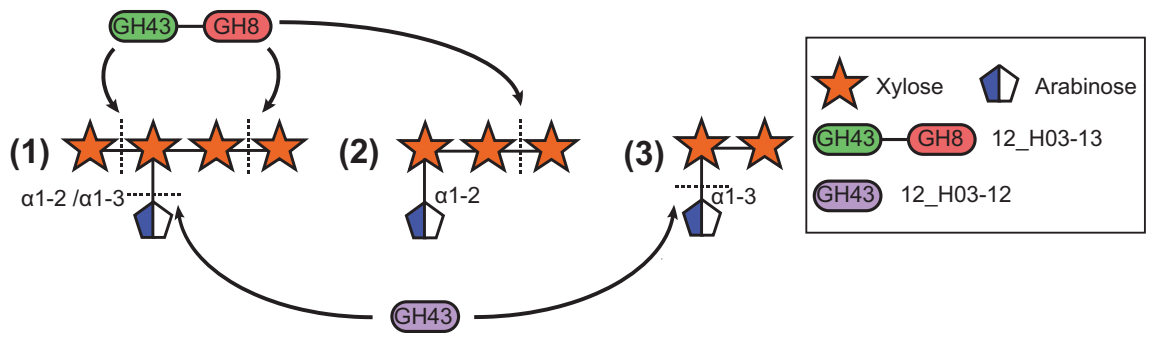

b

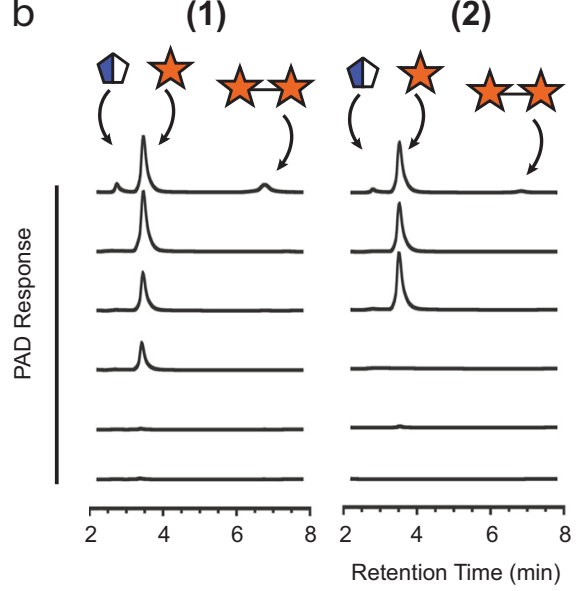

Fig. 3 Activity of GH43s belonging to subfamilies 2 and 7 identified on fosmid 12_H03. a Schematic of the activities of the individual domains of 12_H03-13 and 12_H03-12 on arabinoxylans. These two enzymes were tested for activity on a mixture of $2^{3}-\alpha$-L-arabinofuranosyl-xylotetraose and $3^{3}-\alpha$-L-arabinofuranosyl-xylotetraose $(\mathbf{1}), 2^{3}$ $\alpha$-L-arabinofuranosyl-xylotriose (2) and $3^{2}-\alpha$-L-arabinofuranosylxylobiose (3). The GH8 domain of 12_H03-13 releases xylose from the reducing end of (1) and (2). The GH43 domain of 12_H03-13 releases xylose from the non-reducing end of (1). 12-H03-12, a GH43

$\alpha$-xylosidases) on the three fosmids 09_O03, 12_H03, and 11_K01 leads us to speculate that these fosmids may target fucogalactoxyloglucan which is present in most dicots and gymnosperms [51, 52]

Moreover, we identified 15 fosmids spanning 5 identity groups containing Sus-like genes (SusC or SusD), leading indicators for the identification of PULs using an automated PUL prediction tool [53] (Fig. 4b). Eight of these clones exhibited near complete nucleotide identity (5_O06, 05_O07d，5_O08，5_P05，5_P06，5_P07，5_P08, and 5_P12) and 4 clones shared near complete nucleotide identity specifically in the PUL interval (12_E14, 11_G03, 5_H01, and 9_K06). The remaining 4 clones (4_C03, 9_C22, and 9_G01) contained unique PUL intervals. Representative PULs from each identity group were compared to the RefSeq database to see if they are also found in sequenced microbial genomes. PULs from 9_C22 and 9_G01 exhibited 99\% and 98\% nucleotide identity respectively to distinct regions of the Alistipes senegalensis JC50 genome whereas the most common PUL represented by belonging to subfamily 7 is able to release arabinose from only the oligomers containing an arabinose $\alpha-1$-3-linkage. b High performance anion exchange chromatography with pulsed amperometric detection (HPAEC-PAD) analysis of the degradation of (1), (2) and (3) catalyzed by H03-12, H03-13, H03-13_E209A (GH43 domain mutant, denoted with an X on the GH43 domain) or H03-13_E507A (GH8 domain mutant, denoted with an $\mathrm{X}$ on the GH8 domain) and their combinations

5_P08 exhibited 99\% nucleotide identity to the genome of Alistipes finegoldii DSM17242. The remaining identity groups exhibited less than $7 \%$ nucleotide identity to reference genomes, indicating previously unrecognized architectures. In addition to the fosmids which appear to target hemicelluloses mentioned above, 05_H01 and homologs appear to target pectic polymers as they contain a GH28 (a family containing polygalacturonases) and a GH88 which may target the unsaturated reducing ends generated by pectate lyases. The substrates targeted by the PULs present on fosmids 05_P08, 09_G01, and 09_C22 are not immediately apparent, and further biochemical characterization will be needed to reveal their activity.

Taken together, the PULs and gene clusters identified on fosmids appear to target many of the hemicellulosic components of plant cell walls, including glucuronylxylan, xyloglucan, and pectins, which would be present in hard woods. Some of the polymers which these gene cassettes likely act on, however, are not present in hardwoods, such as mixed-linkage glucans, which are mainly found in 
Fig. 4 Gene organization of putative hemicellulose targeting fosmids and SusC/SusD-like encoding fosmids. a Fosmids with gene clusters that may target the hemicellulosic portion of plant biomass within the beaver diet. b SusC/SusD-like encoding fosmids. Putative glycoside hydrolases and SusC/ SusD-like proteins are colored with the same scheme as Fig. 2. ORFs not annotated as a glycoside hydrolase, SusC-like, or SusD-like, are shown in gray. Fosmids Identical to 5_P08 have been omitted for simplicity

\section{a}

Mixed-Linkage Glucans

04_c03

Xylans

04_C21

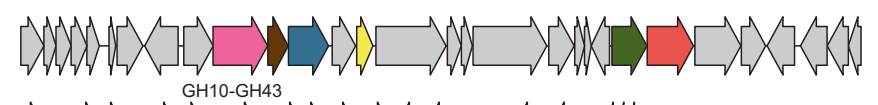

10_G11

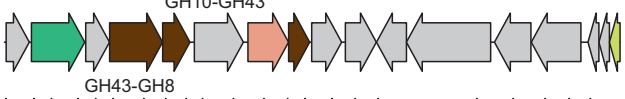

11_G02

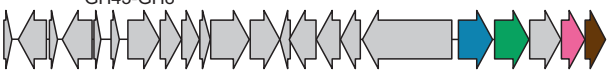

12_H03

11_K01

Xyloglucans

09_003
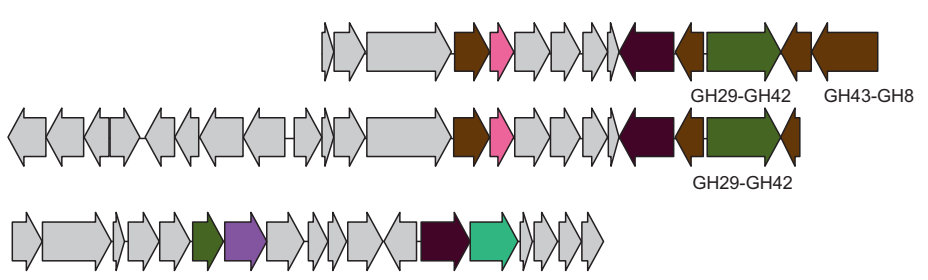

b

04_C03

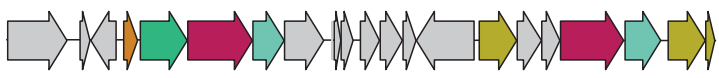

05_P08

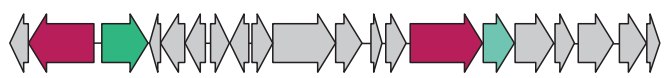

09_G01

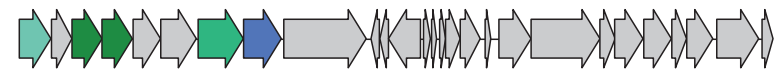

09_C22

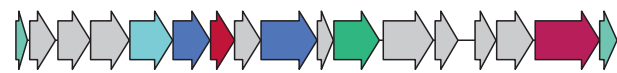

05_H01

11_G03

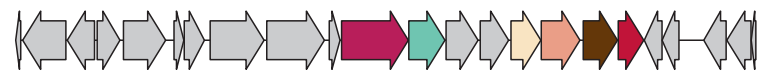

12 E14

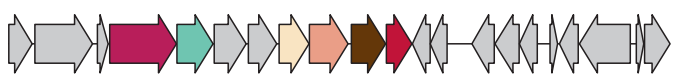

09_K06

$\begin{array}{ll}\square \mathrm{GH} 2 & \square \mathrm{GH} 13 \\ \square \mathrm{GH} 3 & \square \mathrm{GH} 16 \\ \square \mathrm{GH} 10 & \square \mathrm{GH} 25 \\ \square \mathrm{GH} 11 & \square \mathrm{GH} 29\end{array}$

$\mathbb{M} 2$

$\begin{array}{rrrr}\square \text { GH28 } & \square \text { GH36 } & \square \text { GH67 } & \square \text { GH97 } \\ \text { GH31 } & \square \text { GH42 } & \square \text { GH72 } & \square \text { SusD } \\ \square \text { GH32 } & \square \text { GH43 } & \square \text { GH88 } & \square \text { SusC } \\ \square \text { GH35 } & \square \text { GH50 } & \square \text { GH95 } & \square \text { Other }\end{array}$

$5 \mathrm{~kb}$ grasses, and arabinoxylans, which are present in grasses and softwoods. The ability to degrade these polymers may support host nutrition when preferential food sources are scarce. Future characterization of these PULs and gene clusters has potential to shed light on combinatorial biomass deconstruction within the beaver microbiome at the population and community levels of organization [54-57].

Beavers have been described as nature's engineers due to their prolific capacity to reshape forest ecosystems into ponds and meadows, raising groundwater levels and creating new habitats for diverse plants and animals. Beyond their capacity to transform landscapes, beavers are extremely efficient consumers of woody biomass relying on bark, shoots, leaves, and other fibers from hardwood deciduous trees as primary nutritional resources. The current study opens a functional metagenomic window on the capacity of the beaver fecal microbiome to deconstruct woody plants into soluble sugars supporting host nutrition. Although beavers are classified as xylotrophic organisms, their microbiome composition is most similar to those of hindgut-fermenting mammals dominated by Bacteroidetes and Firmicutes. However, the specific strains inhabiting the beaver fecal microbiome harbor abundant and diverse genomic adaptations underlying woody biomass deconstruction, particularly xylan substrates that can be funneled into central metabolism. These adaptations are distributed among and between different populations within the fecal microbiome and encompass multiple GH43 subfamilies (including one containing both GH8 and GH43 domains) that synergize in combination. Several of these subfamilies 
are encoded in PULs representing previously unknown genomic architectures. Targeted conversion screens identified potential substrates for three GH43 subfamilies expanding our mechanistic understanding of this important group of CAZymes. Interestingly we did not identify an abundance of fosmid genes encoding cellulases, indicating the potential for overexpression by specialized strains or compartmentalization of function along the beaver gastrointestinal tract.

The panoply of genes encoding enzymes with hemicellulose degrading activities is presumably required to tackle the complex structures of hardwood biomass in which glucan and xylan backbones are extensively decorated with appended sugars. The presence of two-domain enzymes endows the beaver fecal microbiome with synergistic capacity to efficiently degrade the specific linkages present within the hemicellulose. Not only does this liberate monosaccharides, but the degradation of hemicellulose scaffolds also exposes the underlying cellulose fibers for digestion by the cellulase repertoire, enhancing conversion efficiency. Taken together these results provide a unique perspective on the modular domain architecture and functional specialization driving combinatorial biomass deconstruction in the beaver fecal microbiome. It will be of interest to determine the extent to which beavers combine compartmentalization and sequential biomass deconstruction with combinatorial enzymatic activities acting on lignocellulosic substrates. These deconstruction principles can in turn be used in the design of enhanced enzyme mixtures and whole cell biocatalysts tuned to xylan inputs in modern biorefining ecosystems.

\section{Methods}

\section{Sample collection}

Fecal samples were collected in $50 \mathrm{~mL}$ Falcon tubes on April 12th, 2012 from two beavers that were being cared for at the Critter Care Wildlife Society located in Langley, British Columbia, Canada. Animals were fed branches from a variety of woody plant species, native to the Pacific Northwest. Due to the difficulty of obtaining fresh fecal matter, as beavers defecate underwater, samples were collected from material that had accumulated at the enclosure water outflow grating, within $12 \mathrm{~h}$ of cleaning. The enclosure was open to the environment and not heated. The temperature fluctuated between 7 and $15^{\circ} \mathrm{C}$ in the time before sample collection. As both beavers shared the same enclosure, it was not possible to identify which animal the samples came from. Samples were frozen in a slurry of dry-ice and ethanol and transported to the laboratory on dryice and were stored at $-80^{\circ} \mathrm{C}$.

\section{DNA extraction}

High molecular weight DNA was extracted from four grams of beaver feces as described previously [58]. Extracted DNA was purified by isopropanol precipitation and quantified using the PicoGreen assay (Invitrogen).

\section{PCR amplification of ribosomal SSU rRNA gene sequences}

Following DNA isolation, the V6-V8 region of the small subunit ribosomal RNA (SSU rRNA) gene was PCR amplified with the universal three-domain primers 926F and 1392R containing barcodes for pooled sequencing. Duplicate reactions were pooled and purified and then quantified. Samples were diluted to $10 \mathrm{ng} / \mu \mathrm{L}$ and pooled in equal concentrations. See supplemental methods for further details.

\section{SSU rRNA amplicon sequencing and analysis}

SSU rRNA amplicon pools were sent to the Génome Québec Innovation Centre at McGill University for 454 pyrosequencing using the Roche 454 GS FLX Titanium platform. QIIME [59] was used to quality control and analyze the amplicon sequences, which were then queried against the SILVA database version 111 [20]. See supplementary information for detailed methods.

\section{Metagenomic sequencing and analysis}

Genomic DNA was sent to the Génome Québec Innovation Centre at McGill University for whole genome shotgun sequencing using the Roche 454 GS FLX Titanium platform. 454 sequence data were assembled using MIRA version 4.0.2 [27]. Open reading frames (ORFs) were predicted using Prodigal [60] implemented in the MetaPathways pipeline [28]. The assembled metagenome yielded 151,180 ORFs $>180$ nucleotides in length annotated using LAST [61] implemented in the MetaPathways pipeline based on queries of KEGG [62], COG [63], RefSeq [64], MetaCyc [65], and CAZy [30] databases. Metagenomic data from previous studies $[2,31,32,66]$ were downloaded from the RAST online database and used for comparison.

Metagenomic binning of the MIRA contigs and the 53 assembled fosmid sequences was performed using MaxBin 2.0 [34]. The default MaxBin abundance estimation workflow was unable to estimate abundance of these contigs due to the single-end nature of pyrosequenced reads, therefore abundance files for binning were generated by BWA MEM and a custom script for generating RPKM values in a compatible format [67]. Apart from this, default parameters were used resulting in 30 metagenome-assembled genomes 
(MAGs). All 51 fosmids were binned across 12 different MAGs. MAGs were refined using the Anvi'o metagenomics workflow [35]. Centrifuge was used to assign taxonomy to contigs to assist with manual MAG refinement within this workflow [68]. Additionally, the '--clustercontigs' flag was used to estimate the relatedness of these contigs without abundance information from multiple samples and a minimum contig length of $1,500 \mathrm{bp}$ was enforced to reduce marker gene redundancy from short contigs. checkM was used to evaluate the final completeness and contamination of these MAGs [69], yielding 8 medium-quality (completeness $>50 \%$ and contamination $<10 \%)$ and 22 low-quality MAGs [36]. Taxonomy was assigned to the medium-quality MAGs using Phylosift by taking the deepest taxonomic assignment with $>70 \%$ of the taxonomic annotations [37], see Table S4.

\section{Fosmid library construction and screening}

Genomic DNA was further purified by cesium chloride gradient ultracentrifugation prior to library creation [70]. A large insert library was constructed as described previously [71] using the CopyControl ${ }^{\mathrm{TM}}$ Fosmid Library Production Kit with pCC1FOSTM Vector Kit (EpiCentre). This resulted in a library containing 4608 clones. Functional screening was performed according to procedures by Mewis et al. [42] with modifications. Screening was carried out in phosphate buffer $(25 \mathrm{mM}$ sodium phosphate, $\mathrm{pH}$ 6.0), containing $100 \mu \mathrm{M}$ each of the three fluorogenic substrates (6-chloro-4-methylumbelliferyl $\beta$-cellobioside, 6-chloro-4-methylumbelliferyl $\beta$-xylobioside, and 6-chloro-4-methylumbelliferyl $\beta$-D-xylopyranoside). Screening was performed at a temperature of $37^{\circ} \mathrm{C}$, which is the body temperature of Castor canadensis [72]. Fosmids chosen for sequencing (z-score $>3$ for each substrate) were rearrayed using an automated colonypicking robot (Qpix2, Molecular Devices), into a 96 well plate (Costar 3370) containing $200 \mu \mathrm{L}$ of LB chloramphenicol $(12.5 \mu \mathrm{g} / \mathrm{mL})$ and $10 \%$ glycerol. This master plate was incubated overnight at $37^{\circ} \mathrm{C}$ and then stored at $-80^{\circ} \mathrm{C}$.

The frozen master plate was then used to assay the clones against a panel of eleven different fluorogenic substrates, see supplemental methods for detailed description.

\section{Fosmid sequencing and analysis}

The 96 well master plate was used to inoculate a 96 deepwell plate (Costar) containing $1.65 \mathrm{~mL} \mathrm{LB}$ with chloramphenicol $(12.5 \mu \mathrm{g} / \mathrm{mL})$ and arabinose $(100 \mu \mathrm{g} / \mathrm{mL})$. This plate was incubated with shaking $\left(37^{\circ} \mathrm{C}, 320 \mathrm{rpm}\right)$ for $20 \mathrm{~h}$, then centrifuged at $1,500 \times g$ for $10 \mathrm{~min}$ and the supernatant was decanted. Fosmids were purified from the pelleted cells using a Montage Plasmid MiniprepHTS 96 Kit (Millipore), treated with PlasmidSafe ATP-dependent
DNAse (Epicentre) and quantified using the PicoGreen assay (Invitrogen). Purified DNA was prepared for sequencing on the Illumina MiSeq platform using Nextera XT library preparation kit and 96 sample Nextera V1 index kit. Samples were sequenced using paired end $150 \mathrm{bp}$ reads $(2 \times 150 \mathrm{bp}$ mode). Fosmid sequence quality control and assembly are detailed in the supporting methods. Assembled fosmid contigs were were annotated in the same manner as the assembled metagenome. The resulting RefSeq output files were imported into MEGAN [73] as a way of assigning taxonomy to each fosmid according to the LCAstar algorithm [74].

\section{Cloning and expression of GH43 genes}

One GH43 gene from each of the uncharacterized subfamilies 2, 7, and 28 was chosen for cloning, expression and characterization. The three genes (12_H03-12, 12_H03-13, 12_J03-18), were inserted into a pET28 vector by use of the polymerase incomplete primer extension method [75]. The mutant enzymes H03-13_E507A and H03-13_E209A were produced by means of modified QuikChange mutagenesis [76]. PCR parameters and primers used are detailed in the supplemental methods. The His-tagged proteins were purified by nickel-metal affinity chromatography, as described in the supplemental methods.

\section{GH43 activity assays}

Purified GH43 enzymes were tested against a variety of glycosidase substrates, see supplemental information for detailed methods.

\section{Data accession}

Data is deposited in the NCBI BioProject portal (Bioproject ID: PRJNA261082), for assembled metagenomic reads (BioSample ID: SAMN04122864), unassembled metagenomic reads (BioSample ID: SAMN03389401), functionally identified fosmids (Biosample ID: SAMN03389402), and pyrotags (Biosample ID: SAMN03389403).

Acknowledgements This work was performed under the auspices of the Natural Sciences and Engineering Research Council (NSERC) of Canada, Genome British Columbia, Genome Canada, Canada Foundation for Innovation (CFI), the Tula Foundation funded Centre for Microbial Diversity and Evolution and the Canadian Institute for Advanced Research (CIFAR). ZA and KM were both supported by the NSERC CREATE Genome Sciences and Technology (GSAT) training program at the University of British Columbia. We would like to thank Bernard Henrissat and Nicolas Terrapon for use of PULDB. We would like to thank Rahul Singh and Lindsay Eltis for insightful conversations regarding biomass deconstruction and the future of biorefining. 


\section{Compliance with ethical standards}

Conflict of interest The authors declare that they have no conflict of interest.

Open Access This article is licensed under a Creative Commons Attribution 4.0 International License, which permits use, sharing, adaptation, distribution and reproduction in any medium or format, as long as you give appropriate credit to the original author(s) and the source, provide a link to the Creative Commons license, and indicate if changes were made. The images or other third party material in this article are included in the article's Creative Commons license, unless indicated otherwise in a credit line to the material. If material is not included in the article's Creative Commons license and your intended use is not permitted by statutory regulation or exceeds the permitted use, you will need to obtain permission directly from the copyright holder. To view a copy of this license, visit http://creativecommons. org/licenses/by/4.0/.

\section{References}

1. Martens EC, Kelly AG, Tauzin AS, Brumer H. The devil lies in the details: how variations in polysaccharide fine-structure impact the physiology and evolution of gut microbes. J Mol Biol. 2014;426:3851-65.

2. Muegge BD, Kuczynski J, Knights D, Clemente JC, Gonzalez A, Fontana $\mathrm{L}$, et al. Diet drives convergence in gut microbiome functions across mammalian phylogeny and within humans. Science. 2011;332:970-4.

3. David LA, Maurice CF, Carmody RN, Gootenberg DB, Button JE, Wolfe BE, et al. Diet rapidly and reproducibly alters the human gut microbiome. Nature. 2013;505:559-63.

4. Ley RE, Hamady M, Lozupone C, Turnbaugh PJ, Ramey RR, Bircher JS, et al. Evolution of mammals and their gut microbes. Science. 2008;320:1647-51.

5. Sanders JG, Beichman AC, Roman J, Scott JJ, Emerson D, Mccarthy JJ, et al. Baleen whales host a unique gut microbiome with similarities to both carnivores and herbivores. Nat Commun. 2015;6:8285.

6. Sonnenburg JL, Bäckhed F. Diet-microbiota interactions as moderators of human metabolism. Nature. 2016;535:56-64.

7. Bastien G, Arnal G, Bozonnet S, Laguerre S, Ferreira F, Faure R, et al. Mining for hemicellulases in the fungus-growing termite Pseudacanthotermes militaris using functional metagenomics. Biotechnol Biofuels. 2013;6:78.

8. Hess M, Sczyrba A, Egan R, Kim TW, Chokhawala H, Schroth G, et al. Metagenomic discovery of biomass-degrading genes and genomes from cow rumen. Science. 2011;331:463-7.

9. O'connor RM, Fung JM, Sharp KH, Benner JS, Mcclung C, Cushing S, et al. Gill bacteria enable a novel digestive strategy in a wood-feeding mollusk. Proc Natl Acad Sci USA. 2014;111: E5096-104.

10. Pope PB, Denman SE, Jones M, Tringe SG, Barry K, Malfatti SA, et al. Adaptation to herbivory by the Tammar wallaby includes bacterial and glycoside hydrolase profiles different from other herbivores. Proc Natl Acad Sci. 2010;107:14793-8.

11. Scully ED, Geib SM, Hoover K, Tien M, Tringe SG, Barry KW, et al. Metagenomic profiling reveals lignocellulose degrading system in a microbial community associated with a wood-feeding beetle. PLoS ONE. 2013;8:e73827.

12. Warnecke F, Luginbuhl P, Ivanova N, Ghassemian M, Richardson $\mathrm{TH}$, Stege JT, et al. Metagenomic and functional analysis of hindgut microbiota of a wood-feeding higher termite. Nature. 2007;450:560-5.
13. Armstrong Z, Mewis K, Strachan C, Hallam SJ. Biocatalysts for biomass deconstruction from environmental genomics. Curr Opin Chem Biol. 2015;29:18-25.

14. Busher PE. Food caching behavior of beavers (Castor canadensis): selection and use of woody species. Am Midl Nat. 1996;135: 343-8.

15. Vispo C, Hume ID. The digestive tract and digestive function in the North American porcupine and beaver. Can J Zool. 1995;73: 967-74.

16. Willför S, Sundberg A, Pranovich A, Holmbom B. Polysaccharides in some industrially important hardwood species. Wood Sci Technol. 2005;39:601-17.

17. Currier A, Kitts WD, I. C. Cellulose digestion in the beaver (Castor canadensis). Can J Zool. 1960;38:1109-16.

18. Gruninger RJ, Mcallister TA, Forster RJ. Bacterial and archaeal diversity in the gastrointestinal tract of the north american beaver (Castor canadensis). PLoS ONE. 2016;11:e0156457.

19. Nicholson JK, Holmes E, Kinross J, Burcelin R, Gibson G, Jia W, et al. Host-gut microbiota metabolic interactions. Science. 2012;336:1262-7.

20. Quast C, Pruesse E, Yilmaz P, Gerken J, Schweer T, Yarza P, et al. The SILVA ribosomal RNA gene database project: improved data processing and web-based tools. Nucl. Acids Res. 2013;41:D590-D596.

21. Horn S, Durka W, Wolf R, Ermala A, Stubbe A, Stubbe M, et al. Mitochondrial genomes reveal slow rates of molecular evolution and the timing of speciation in beavers (Castor), one of the largest rodent species. PLoS ONE. 2011;6:e14622.

22. Cotta M., Forster R. (2006) The Family Lachnospiraceae, Including the Genera Butyrivibrio, Lachnospira and Roseburia. In: Dworkin M., Falkow S., Rosenberg E., Schleifer KH., Stackebrandt E. (eds) The Prokaryotes. Springer, New York, NY, pages $1002-1021$.

23. Wong, MT, Wang, W, Lacourt, M, Couturier, M, Edwards, EA \& Master, ER (2016). Substrate-driven convergence of the microbial community in lignocellulose-amended enrichments of gut microflora from the Canadian beaver (Castor canadensis) and North American Moose (Alces americanus). Front. Microbiol. 7:961.

24. Favia G, Weldon L, Abolins S, Lenzi L, Bourne C, Riley EM, et al. The gut microbiota of wild mice. PLoS ONE. 2015;10: e0134643.

25. Maurice CF, Cl Knowles S, Ladau J, Pollard KS, Fenton A, Pedersen $\mathrm{AB}$, et al. Marked seasonal variation in the wild mouse gut microbiota. ISME J. 2015;9:2423-34.

26. Schmieder R, Edwards R. Quality control and preprocessing of metagenomic datasets. Bioinformatics. 2011;27:863-4.

27. Chevreux B, Pfisterer T, Drescher B, Driesel AJ, Müller WEG, Wetter $\mathrm{T}$, et al. Using the miraEST assembler for reliable and automated mRNA transcript assembly and SNP detection in sequenced ESTs. Genome Res. 2004;14:1147-59.

28. Konwar KM, Hanson NW, Bhatia MP, Kim D, Wu SJ, Hahn AS, et al. MetaPathwaysv2.5: quantitative functional, taxonomic and usability improvements. Bioinformatics. 2015;31:3345-7.

29. Kumar PS, Brooker MR, Dowd SE, Camerlengo T. Target region selection is a critical determinant of community fingerprints generated by $16 \mathrm{~S}$ pyrosequencing. PLoS ONE. 2011;6:e20956.

30. Lombard V, Golaconda Ramulu H, Drula E, Coutinho PM, Henrissat B. The carbohydrate-active enzymes database (CAZy) in 2013. Nucl Acids Res. 2014;42:D490-5.

31. Pope PB, Mackenzie AK, Gregor I, Smith W, Sundset MA, Mchardy AC, et al. Metagenomics of the svalbard reindeer rumen microbiome reveals abundance of polysaccharide utilization loci. PLoS ONE. 2012;7:e38571. 
32. Zhu L, Wu Q, Dai J, Zhang S, Wei F. Evidence of cellulose metabolism by the giant panda gut microbiome. Proc Natl Acad Sci. 2011;108:17714-9.

33. Mcmurdie PJ, Holmes S. Waste not, want not: why rarefying microbiome data is inadmissible. PLoS Comput Biol. 2014;10: e1003531.

34. Wu, YW, Simmons, BA \& Singer, SW (2015). MaxBin 2.0: an automated binning algorithm to recover genomes from multiple metagenomic datasets. Bioinformatics. 32;4:605-7.

35. Eren AM, Esen OC, Quince C, Vineis JH, Morrison HG, Sogin ML, et al. Anvi'o: an advanced analysis and visualization platform for 'omics data. PeerJ. 2015;3:e1319.

36. Bowers RM, Kyrpides NC, Stepanauskas R, Harmon-Smith M, Doud D, Reddy TBK, et al. Minimum information about a single amplified genome (MISAG) and a metagenome-assembled genome (MIMAG) of bacteria and archaea. Nat Biotechnol. 2017;35:725-31.

37. Darling AE, Jospin G, Lowe E, Matsen FAT, Bik HM, Eisen JA. PhyloSift: phylogenetic analysis of genomes and metagenomes. PeerJ. 2014;2:e243.

38. Louis P, Duncan SH, Mccrae SI, Millar J, Jackson MS, Flint HJ. Restricted distribution of the butyrate kinase pathway among butyrate-producing bacteria from the human colon. J Bacteriol. 2004;186:2099-106.

39. Salyers AA, West SE, Vercellotti JR, Wilkins TD. Fermentation of mucins and plant polysaccharides by anaerobic bacteria from the human colon. Appl Environ Microbiol. 1977;34:529-33.

40. Ndeh D, Rogowski A, Cartmell A, Luis AS, Baslé A, Gray J, et al. Complex pectin metabolism by gut bacteria reveals novel catalytic functions. Nature. 2017;544:65-70.

41. Mewis K, Armstrong Z, Song YC, Baldwin SA, Withers SG, Hallam SJ. Biomining active cellulases from a mining bioremediation system. J Biotechnol. 2013;167:462-71.

42. Mewis, K, Taupp, M \& Hallam, SJ (2011). A high throughput screen for biomining cellulase activity from metagenomic libraries. J Vis Exp. 48:2461.

43. Chen HM, Armstrong Z, Hallam SJ, Withers SG. Synthesis and evaluation of a series of 6-chloro-4-methylumbelliferyl glycosides as fluorogenic reagents for screening metagenomic libraries for glycosidase activity. Carbohydr Res. 2016;421:33-9.

44. Fontes CMGA, Gilbert HJ. Cellulosomes: highly efficient nanomachines designed to deconstruct plant cell wall complex carbohydrates. Annu Rev Biochem. 2010;79:655-81.

45. Martens EC, Koropatkin NM, Smith TJ, Gordon JI. Complex glycan catabolism by the human gut microbiota: the bacteroidetes sus-like paradigm. J Biol Chem. 2009;284:24673-7.

46. Simpson JT, Wong K, Jackman SD, Schein JE, Jones SJM, Birol FNß. ABySS: a parallel assembler for short read sequence data. Genome Res. 2009;19:1117-23.

47. Mewis K, Lenfant N, Lombard V, Henrissat B. Dividing the large glycoside hydrolase family 43 into subfamilies: a motivation for detailed enzyme characterization. Appl Environ Microbiol. 2016; 82:1686-92.

48. Brunecky R, Alahuhta M, Xu Q, Donohoe BS, Crowley MF, Kataeva IA, et al. Revealing nature's cellulase diversity: the digestion mechanism of Caldicellulosiruptor bescii CelA. Science. 2013;342:1513-6.

49. Tamura K, Hemsworth GR, Déjean G, Rogers TE, Pudlo NA, Urs $\mathrm{K}$, et al. Molecular mechanism by which prominent human gut bacteroidetes utilize mixed-linkage beta-glucans, major healthpromoting cereal polysaccharides. Cell Rep. 2017;21:417-30.

50. Wang, K, Pereira, GV, Cavalcante, JJV, Zhang, M, Mackie, R \& Cann, I (2016). Bacteroides intestinalis DSM 17393, a member of the human colonic microbiome, upregulates multiple endoxylanases during growth on xylan. Scientific Reports, 6 .
51. Hsieh YSY, Harris PJ. Structures of xyloglucans in primary cell walls of gymnosperms, monilophytes (ferns sensu lato) and lycophytes. Phytochemistry. 2012;79:87-101.

52. Pauly M, Keegstra K. Biosynthesis of the plant cell wall matrix polysaccharide xyloglucan. Annu Rev Plant Biol. 2016;67: 235-59.

53. Terrapon N, Lombard V, Gilbert HJ, Henrissat B. Automatic prediction of polysaccharide utilization loci in Bacteroidetes species. Bioinformatics. 2015;31:647-55.

54. Cuskin F, Lowe EC, Temple MJ, Zhu Y, Cameron EA, Pudlo NA, et al. Human gut Bacteroidetes can utilize yeast mannan through a selfish mechanism. Nature. 2015;517:165-9.

55. Larsbrink J, Rogers TE, Hemsworth GR, Mckee LS, Tauzin AS, Spadiut $\mathrm{O}$, et al. A discrete genetic locus confers xyloglucan metabolism in select human gut Bacteroidetes. Nature. 2014a;506: 498-502.

56. Larsbrink J, Thompson AJ, Lundqvist M, Gardner JG, Davies GJ, Brumer $\mathrm{H}$. A complex gene locus enables xyloglucan utilization in the model saprophyte Cellvibrio japonicus. Mol Microbiol. 2014b;94:418-33.

57. Rogowski A, Briggs JA, Mortimer JC, Tryfona T, Terrapon N, Lowe EC, et al. Glycan complexity dictates microbial resource allocation in the large intestine. Nat Commun. 2015;6: 7481.

58. Lee S, Hallam SJ. Extraction of High Molecular Weight Genomic DNA from Soils and Sediments. Journal of Visualized Experiments : JoVE. 2009;(33):1569. doi:10.3791/1569.

59. Caporaso JG, Kuczynski J, Stombaugh J, Bittinger K, Bushman FD, Costello EK, et al. QIIME allows analysis of high-throughput community sequencing data. Nat Meth. 2010;7: $335-6$.

60. Hyatt D, Chen GL, Locascio PF, Land ML, Larimer FW, Hauser LJ. Prodigal: prokaryotic gene recognition and translation initiation site identification. BMC Bioinforma. 2010;11:119.

61. Kielbasa SM, Wan R, Sato K, Horton P, Frith MC. Adaptive seeds tame genomic sequence comparison. Genome Res. 2011;21: 487-93.

62. Kanehisa M, Goto S. KEGG: kyoto encyclopedia of genes and genomes. Nucl Acids Res. 2000;28:27-30.

63. Tatusov RL, Natale DA, Garkavtsev IV, Tatusova TA, Shankavaram UT, Rao BS, et al. The COG database: new developments in phylogenetic classification of proteins from complete genomes. Nucl Acids Res. 2001;29:22-8.

64. Pruitt KD, Maglott DR. RefSeq and LocusLink: NCBI genecentered resources. Nucl Acids Res. 2001;29:137-40.

65. Caspi R, Altman T, Dreher K, Fulcher CA, Subhraveti P, Keseler IM, et al. The MetaCyc database of metabolic pathways and enzymes and the BioCyc collection of pathway/genome databases. Nucl Acids Res. 2012;40:D742-53.

66. Swanson KS, Dowd SE, Suchodolski JS, Middelbos IS, Vester BM, Barry KA, et al. Phylogenetic and gene-centric metagenomics of the canine intestinal microbiome reveals similarities with humans and mice. ISME J. 2010;5:639-49.

67. Li, H 2013. Aligning sequence reads, clone sequences and assembly contigs with BWA-MEM. ArXiv e-prints [Online]. http://arxiv.org/abs/1303.3997

68. Kim D, Song L, Breitwieser FP, Salzberg SL. Centrifuge: rapid and sensitive classification of metagenomic sequences. Genome Res. 2016;26:1721-9.

69. Parks DH, Imelfort M, Skennerton CT, Hugenholtz P, Tyson GW. CheckM: assessing the quality of microbial genomes recovered from isolates, single cells, and metagenomes. Genome Res. 2015;25:1043-55

70. Wright JJ, Lee S, Zaikova E, Walsh DA, Hallam SJ. DNA Extraction from $0.22 \mu \mathrm{M}$ Sterivex Filters and Cesium Chloride 
Density Gradient Centrifugation. Journal of Visualized Experiments : JoVE. 2009;(31):1352. doi:10.3791/1352.

71. Taupp M, Lee S, Hawley A, Yang J, Hallam SJ. Large Insert Environmental Genomic Library Production. Journal of Visualized Experiments : JoVE. 2009;(31):1387. doi:10.3791/1387.

72. Dyck AP, Macarthur RA. Seasonal patterns of body-temperature and activity in free-ranging beaver (Castor-Canadensis). Can J Zool-Rev Can De Zool. 1992;70:1668-72.

73. Huson DH, Weber N. Microbial community analysis using MEGAN. Methods Enzymol. 2013;531:465-85.
74. Hanson, NW, Konwar, KM \& Hallam, SJ (2016). LCA*: an entropy-based measure for taxonomic assignment within assembled metagenomes. Bioinformatics.

75. Klock HE, Lesley SA. The polymerase incomplete primer extension (PIPE) method applied to high-throughput cloning and site-directed mutagenesis. Methods Mol Biol. 2009;498:91-103.

76. Kokoska RJ, Mcculloch SD, Kunkel TA. The efficiency and specificity of apurinic/apyrimidinic site bypass by human DNA polymerase eta and Sulfolobus solfataricus Dpo4. J Biol Chem. 2003;278:50537-45. 